\title{
A Framework for Complex Cloud-Integrated CPSs
}

\author{
Qiuxia Yang ${ }^{\text {a) }}$ and Lichen Zhang ${ }^{\text {b) }}$ \\ Faculty of Computer Science and Technology Guangdong University of Technology Guangzhou 510090, China \\ a) Qiuxia Yang: yqx1415@163.com \\ b)LichenZhang@zhanglichen1962.com

\begin{abstract}
The emerging Cyber Physical Systems (CPSs) are complex, muliti-disciplinary next generation engineered systems that integrate embedded computing technology into the physical phenomena by using transformation research approaches. A cloud computing environment offers a simplified, centralized platform or resources for use when needed at a low cost. One of the key functionalities of this type of computing is to allocate the resources on an individual demand. Cloud-integrated CPS(CCPS) will open the door to allow previously unachievable application scenarios to build, deployed, managed and controlled effectively. Inspired by the concept of Micro-Service, we propose a novel architecture for Cloud-Integrated cyber physical system (termed MSCCPSA). Then, we dissent three potential challenges and provide solutions from the perceive of micro service, including the virtualized resource management techniques, the scheduling techniques, and life cycle management. The proposed architecture aims at a holistic view with increased adaptability, where the controllers efficiently collaborate to quickly capture and respond to abnormal situations in self adjusting manner. The proposed framework architecture can help Cloud-Integrated Cyber Physical Systems study.
\end{abstract}

Key words: Cyber Physical Systems (CPSs); Micro Service; Cloud Computing; framework.

\section{INTRODUCTION}

Cyber Physical Systems (CPSs) [1] is an emerging area, and it was coined at the National Science Foundation(NSF) in the United States around 2006[2]. The Cyber Physical Systems (CPSs) are integrations of monitoring, communication and computation operation. These systems can capture physical data usually by using the control of embedded computers and networks monitors, and response to the environment by using actuators and software components. There are lots of sensor data generating and operating in the interaction process. CPSs are very complex heterogeneous network systems, science their architectures must support the integration of various heterogeneous components. Nowadays, CPSs are widely used in the traffic control systems [3], high confidence medical systems, process control systems, advanced automotive systems, avionics systems, defense systems, distributed robotics, critical infrastructure control systems, manufacturing and so on.

The standard definition of Cloud computing by NIST (National Institute of Standards and Technology) [4] as" a model for enabling convenient, on-demand network access to a shared pool of configurable computing resources (e.g., networks, server, storage, application, and services) that can be rapidly provisioned and released with minimal management effort or service provider interaction". Cloud computing can offer the IT resource capabilities as a service and provide an effective solution for addressing some problems arising from CPSs. The could computing infrastructure can effectively support the collection, storage and processing of a large number of distributed heterogeneous data. Meanwhile, cloud computing can provide a unified platform that can support CPSs requirements, including the hierarchical structure, information management, and security management etc. Science the support of the cloud platform and cloud service application, CPSs will promote the efficiency of the system, increase the quality of service, and improve the friendliness of environment, particularly in a big data environment.

Monolithic applications already successful apply to the most software systems, but it harder to changes a problem that ought to only affect one module within other module. The monolithic cloud applications are usually 
deployed as a single package on a web container. These are easily to deploy and to develop for non-experienced developers. However, the monolithic systems are not easily scalable and rebuilt, especially more applications are being deployed to the cloud. To address these issues, some new architectural style, such as MSA, have been proposed in the last years.

Micro Services Architecture (MSA) [5] offers an important architectural style by their decomposable nature. Service Oriented Architecture (SOA) approach is now widely adoption, and it has matured and feasible design principles [6]. SOA as a lightweight method, and it provides a shared service about specific-purpose implementation [7]. Meanwhile, it also provides service composition, service reuse and infrastructure efficiency. SOA is widely used because it promoted the interoperability and reusability of legacy components. However, there are some related problems exiting from the existing SOA [8]. MSA has a lot of different properties that including elastic, isolated, resilient, autonomous, and responsive. The most of these properties are different from the previous service-oriented approaches. MSA now provides a new way to think about structuring applications.

In this paper, we propose the simplified architecture and corresponding technologies in the design of CCPS for MSA (terms as MSA-CCPS), review several challenges and provide potential solutions to improve the Quality of Service (QoS) of the related MSA-CCPS. The remainder of this paper is organized as follows: The section 2 presents the MSA-CCPS and its corresponding technologies in detail. Section 3 discusses key technology challenges for MSA-CCPS and corresponding solutions. Finally, we present some conclusions.

\section{ARCHITECTURE OF MICRO SERVICE-ORIENTED CLOUD-INTEGRATED}

The system of cloud-Integrated CPSs has existed in many domains, and some corresponding research has made significant achievements recently. CCPSs can combine communication and computation together with physical space [9]. The key ICT technologies of MSA play an important role in the sustainability of cloud-integrated cyber physical systems, and it involves the integration of the physical world and computational world. MSA composed from a lot of independently deployable services that offers a particular way to design software application of CCPSs. MSA's advantages makes it suitable for a growing scale of cyber physical systems, and MSA is the right approach for implementation of CCPSs. With the perfection of cloud infrastructure service platform, the adoption of MSA will make CCPSs automation more easily. The sensing and actuation capabilities and information integration flexibility will be exposed in the MSA approach. The high-level services are made up of the basic micro services that including the sensing and actuation capabilities of devices for data processing and decision-making services.

With the cloud service platform, we can easily analyze and storage data, exchange information among CCPSs by using the big data and cloud computing technologies. But we must think about several questions, such as how to divided the size of micro services, find the demands of CCPSs etc.

In the process of implement of MSA-CCPSs, we can divide it as three steps. Firstly, we need to deploy the basic infrastructure to make the equipment to communicate each other. Secondly, the architecture can supply platform for processing data and information. The last is to provide various application on CPSs to promote service efficiency and performance. MSA on cloud can provide a flexible, efficient, and scalable method build applications as suites of services and offer a solution for the user demands of high quality.

In the near future, services will be the core resources of CCPSs. The architecture of MAS-CCPSs is an approach to developing CCPSs's application as a suite of small services, each service has its own process and communicates with lightweight mechanisms. These services can have built sensing and actuating capabilities and independently deploy with automated deployment machinery. It may be written in different programming language and use different data storage technologies or may be managed by different teams.

The MAS technologies are very important to strength the functionality and performance of the whole CCPSs. Although many apply have been made on MSA, there are still many new issues and challenges in the context of CCPSs we will state the challenges and possible solutions in the next section.

\section{CHALLENGES AND POSSIBLE SOLUTIONS FOR MSA IN THE CCPSS}

As mentioned, MSA is a useful architecture with its inherent features, such as decomposable, resilient, isolated, autonomous, and responsive etc. At present cloud computing systems cannot often meet the application requirement of MSA. To overcome to this problem, it needs to develop efficient virtualization technologies to improve resource management. With some innovative services and decisions are provided by designing CCPSs application. Therefore, it is imperative to combine the emerging fields of cloud computing and big data technology. 
The architecture designs of MSA Cloud-based is a challenge, which require the reconstruction of all layers and components in the CCPSs. CCPSs can adopt MSA architectural styles and need to adapt some standards and practices by adding extra overhead at the design phase. These small and autonomous services of CCPSs are deployed independently, and they can scale easily and independently other services that is more suitable for the user's needs. These can maintain more fault tolerant from a failure in these services that does not led to the whole system broke down.

The architecture of MSA is complex to design, the service reusability, services cohesion, service granularity of MSA about CCPS will be considered in the future.

\section{CONCLUSION}

This paper presented a micro service-oriented architecture name MSA for Cloud-Integrated cyber physical system (termed MSCCPSA) that address semantic integration of heterogeneous data sources about sustainable projects. This domain has an interdisciplinary perspective that combines environmental, social and economic information. The complexity involved has demanded specific functionalities to support the decision-making process in this context.

\section{REFERENCES}

1. E. A. Lee, "CPS foundations," in Proc. 47th Des. Autom. Conf., New York,NY, USA: ACM, 2010, pp. 737742.

2. Edward A. Lee and Sanjit A. Seshia, "Introduction to Embedded Systems: A Cyber-Physical Systems Approach", 1st Edition, 2011.

3. D. Ni et al., "Cyber-Physical Integration to Connect Vehicles for Transformed Transportation Safety and Efficiency," Proc. 25th Int'1. Conf. Industrial Eng. And Other Applications of Applied Intelligent Sys., Dalian,China, 2012, pp. 88-94.

4. Kuyoro S. O.; Ibikunle F; \& Awodele O., "Cloud Computing Security Issues and challenges" in Proceeding of International Journal of Computer Networks (IJCN), Volume (3), Issue (5), 2011.

5. https://martinfowler.com/microservices/

6. Thomas Erl. SOA Principles of Service Design (The Prentice Hall Service-Oriented Computing Series from Thomas Erl). Prentice Hall PTR, Upper Saddle River, NJ, USA, 2007

7. A. Arsanjani, G. Booch, T. Boubez, P. Brown, D. Chappell, J. deVadoss, T. Erl, N. Josuttis, D. Krafzig, M. Little, and others, "The soa manifesto," SOA Manifesto, Oct., 2009

8. https://martinfowler.com/bliki/ServiceOrientedAmbiguity.html

9. E. Simmon et al, "A Vision of Cyber-Physical Cloud Computing for Smart Networked Systems," NIST, [Online] Available: http://www.nict.go.jp/en/univ-com/isp/research-cpcc.html 